University of Warwick institutional repository: http://go.warwick.ac.uk/wrap This paper is made available online in accordance with publisher policies. Please scroll down to view the document itself. Please refer to the repository record for this item and our policy information available from the repository home page for further information.

To see the final version of this paper please visit the publisher's website. Access to the published version may require a subscription.

Author(s): J. SAI-LAP LAM and DAVID G. DRITSCHEL Article Title: On the beta-drift of an initially circular vortex patch Year of publication: 2001 Link to published version: http://dx.doi.org/10.1017/S0022112001003974 Publisher statement: None 


\title{
On the beta-drift of an initially circular vortex patch
}

\author{
By J. SAI-LAP LAM ${ }^{1} \dagger$ AND DAVID G. DRITSCHEL ${ }^{2} \ddagger$ \\ ${ }^{1}$ Department of Applied Mathematics and Theoretical Physics, University of Cambridge, \\ Silver Street, Cambridge CB3 9EW, UK \\ ${ }^{2}$ Department of Mathematics, University of Warwick, Coventry CV4 7AL, UK
}

(Received 25 November 1997 and in revised form 27 July 2000)

The nonlinear inviscid evolution of a vortex patch in a single-layer quasi-geostrophic fluid and within a background planetary vorticity gradient is examined numerically at unprecedented spatial resolution. The evolution is governed by two dimensionless parameters: the initial size (radius) of the vortex compared to the Rossby deformation radius, and the initial strength of the vortex compared to the variation of the planetary vorticity across the vortex. It is found that the zonal speed of a vortex increases with its strength. However, the meridional speed reaches a maximum at intermediate vortex strengths. Both large and weak vortices are readily deformed, often into elliptical and tripolar shapes. This deformation is shown to be related to an instability of the instantaneous vorticity distribution in the absence of the planetary vorticity gradient $\beta$.

The extremely high numerical resolution employed reveals a striking feature of the flow evolution, namely the generation of very sharp vorticity gradients surrounding the vortex and extending downstream of it in time. These gradients form as the vortex forces background planetary vorticity contours out of its way as it propagates. The contours close to the vortex swirl rapidly around the vortex and homogenize, but at some critical distance the swirl is not strong enough and, instead, a sharp vorticity gradient forms. The region inside this sharp gradient is called the 'trapped zone', though it shrinks slowly in time and leaks. This leaking occurs in a narrow wake called the 'trailing front', another zone of sharp vorticity gradients, extending behind the vortex.

\section{Introduction}

Isolated coherent vortices are ubiquitous in planetary atmospheres, in the oceans and, generally, in rotating, stratified fluids. One of the most striking examples is Jupiter's Great Red Spot. These vortices appear in various forms, but are most frequently monopolar or dipolar. They are characterized by their longevity (lifetimes greatly exceeding their characteristic rotation time) and their ability to withstand the background turbulent fluctuations ever present in real flows. An important consequence is that vortices tend to trap and advect fluid particles over lengths much greater than their characteristic size. For vortex dipoles, such non-diffusive tracer transport is enabled by the mutual propagation of the constituent vortex pair while, for monopolar vortices, it may be enabled by an ambient potential vorticity gradient.

$\dagger$ Present address: Department of Mathematics, Hong Kong University of Science and Technology, Clear Water Bay, Hong Kong.

$\mp$ Present address: Mathematical Institute, University of St Andrews, North Haugh, St Andrews KY16 9SS, Fife, Scotland, UK. 
In the oceans, for example, monopolar vortices called Gulf Stream rings are formed from the meandering of the Gulf Stream (Fuglister \& Worthington 1951). These vortices largely retain their distinctive thermal, chemical and biological contents, and as such have considerable influence on the surrounding environment. Here, in this paper, we focus on the propagation and stability of these monopolar vortices within an idealized background planetary vorticity gradient, namely a $\beta$-plane.

Numerous studies of this topic have been made in the past few decades. Approaches include theoretical (Adem 1956; Flierl 1977; Sutyrin 1987, 1988; Reznik 1990, 1992; Sutyrin \& Flierl 1994; Sutyrin \& Morel 1997), numerical (McWilliams \& Flierl 1979; Meid \& Lindemann 1979; Sutyrin et al. 1994) and experimental (Firing \& Beardsley 1976; Carnevale, Kloosterziel \& van Heijst 1991). Extensive discussions of vortex drift on a $\beta$-plane and the associated Rossby wave radiation can also be found in the past literature (see e.g. Sutyrin 1987, 1988 and McDonald 1998). Nonetheless, a full understanding of the nonlinear flow development up to late times is lacking in our view, and it appears to be beyond analytical treatment.

The purpose of the present work is to provide an accurate picture of this late and complex stage of evolution, and to point out its most salient characteristics. We shall study the circular vortex patch model which, arguably the simplest conceptually, has not yet been adequately examined owing to technical difficulties arising from the vorticity discontinuity at the vortex edge. Sutyrin \& Flierl (1994) have examined the short-time behaviour of this model analytically; here we shall present high-resolution numerical results for the long-term behaviour.

The dynamics of an isolated vortex on a $\beta$-plane is governed by two inter-related processes: (i) the generation of a residual (or regular) flow by the redistribution of ambient potential vorticity within the planetary vorticity gradient, and (ii) the deformation of the vortex (cf. Sutyrin \& Flierl 1994). Using a vortex patch model, one can track the detailed movement and deformation of the vortex boundary without encountering the difficulty and ambiguity intrinsic to Gaussian or other (continuously) distributed vortices. The vortex patch model allows one to deduce straightforwardly the flow arising from the vortex and thus to dissociate these two processes for separate analysis.

Owing to the nonlinearity of the governing equations, the long-term evolution of a vortex patch can only be treated by direct numerical simulation. However, in general it is difficult to simulate flows with vorticity discontinuities, since most numerical methods depend on down-gradient diffusion of some sort for stability. Contour dynamics (CD, see Dritschel 1989), which seems to be the only appropriate scheme for this type of problem, is not satisfactory either. The reason lies in its lack of efficiency when a multitude of contours are followed - this is the case for $\beta$-plane geometry as we shall see shortly. As a consequence, there has been little previous work on the long-term behaviour of vortex patches on a $\beta$-plane. Perhaps the most closely related work is that of Sutyrin \& Morel (1997), who studied this problem both analytically and numerically. For their numerical simulations, they used a hyperviscous pseudo-spectral method, which compromised the long-time accuracy of their solutions. In particular, the sharp gradients at the vortex boundary, and those created during the vortex evolution, were not well resolved.

Here, we re-examine this problem numerically using a hybrid numerical algorithm, the contour-advective semi-Lagrangian (CASL) algorithm (Dritschel \& Ambaum 1997). This algorithm combines the most efficient and accurate aspects of CD and the pseudo-spectral method, leading to a huge gain in efficiency for a given accuracy.

The purpose of this paper is two-fold: first, to describe the CASL application to $\beta$-plane geometry, and second, to study the long-time behaviour of initially circular 
vortex patches with different sizes and strengths on the $\beta$-plane. The formulation of the model problem, to be investigated in the context of the equivalent barotropic quasi-geostrophic $(\mathrm{QG})$ approximation, is introduced in $\S 2$. A decomposition of the whole flow field into regular and singular parts is described in the second half of this section. These two parts are respectively related to the induced residual flow generated by the redistribution of ambient potential vorticity and by the deformation of the vortex. Details of the numerical implementation of the CASL algorithm for $\beta$-plane geometry are discussed in $\S 3$. There, the evolution of the potential vorticity field is illustrated in several representative cases. Many well-known features, such as tripolar structures and the trailing front, are here obtained at extraordinarily high resolution. In $\S 4$, we present quantitative results for the vortex trajectories, while in $\S 5$ we examine the vortex deformation and its relation to the stability of the local vorticity distribution. In $\S 6$, we focus on the development of sharp potential vorticity gradients at the periphery of the trapped zone and in the 'trailing front' or wake of the vortex. Our conclusions are presented in $\$ 7$.

\section{Mathematical formulation}

\subsection{Model problem}

We examine the evolution of an initially circular vortex patch moving on a $\beta$-plane. Let $\beta$ be the planetary vorticity gradient, $R_{d}$ the Rossby deformation radius and $\psi$ the streamfunction. The equivalent barotropic QG equation (Pedlosky 1987), describing the material conservation of QG potential vorticity (denoted as PV hereinafter) in a single-layer shallow-water fluid is given by

$$
\frac{\mathrm{D} q}{\mathrm{D} t} \equiv \frac{\partial q}{\partial t}+J(\psi, q)=0,
$$

where

$$
q=\omega+\beta y, \quad \omega=\left(\nabla^{2}-R_{d}^{-2}\right) \psi .
$$

$J(.,$.$) and \nabla^{2}$ are the Jacobian and horizontal Laplacian operators respectively. The horizontal flow $\boldsymbol{u} \equiv(u, v)$ is non-divergent and thus can be expressed as

$$
u=-\frac{\partial \psi}{\partial y}, \quad v=\frac{\partial \psi}{\partial x} .
$$

Cartesian coordinates are adopted with positive $x$ eastward and positive $y$ northward. Note that the PV drives the entire flow evolution; its instantaneous distribution wholly determines the velocity field, and this field advects the PV to the next instant of time. Although this ideal property does not carry over in its entirety to the unapproximated equations governing atmospheric and oceanic motions, it is often an excellent approximation (see Hoskins, McIntyre \& Robertson 1985). The real limitation of the present model is the use of just one layer.

The initial condition considered consists of a circular vortex patch with uniform PV anomaly:

$$
\left.\omega(x, y)\right|_{t=0}= \begin{cases}\omega_{0}=\text { constant } & \text { if } \sqrt{x^{2}+y^{2}}<R \\ 0 & \text { otherwise }\end{cases}
$$

where $R$ denotes its radius. Apart from the limiting cases $R_{d}=0$ or $\beta=0$, we may 
take, without loss of generality,

$$
\beta=1, \quad R_{d}=1
$$

The total number of parameters is thus reduced to two: $\omega_{0}$ and $R$. Equations (2.1)(2.5) constitute the complete mathematical description of our model problem. The evolution for other values of $\beta$ and $R_{d}$ can be related to this model form by a simple rescaling on the characteristic Rossby wave time and length scales:

$$
T=\left(\beta R_{d}\right)^{-1}, \quad L=R_{d},
$$

which leads to the following relationships:

$$
\omega_{0}=\frac{\omega_{0}^{*}}{\beta R_{d}}, \quad R=\frac{R^{*}}{R_{d}},
$$

between the original parameters (marked with an asterisk) and the model parameters. Note that there are two different intrinsic time scales in this problem. One is the fast time scale associated with the swirling velocity field due to the PV anomaly $4 \pi / \omega_{0}$. The other is the slow time scale associated with the Rossby wave propagation $T$. The latter time scale characterizes the propagation of the vortex, and here we consider $t \gg T$.

\subsection{Flow decomposition}

We decompose the flow as follows:

$$
q=q_{s}+q_{r}, \quad \psi=\psi_{s}+\psi_{r}
$$

where

$$
q_{s}=\left(\nabla^{2}-1\right) \psi_{s}, \quad q_{r}=\left(\nabla^{2}-1\right) \psi_{r}+y .
$$

The quantity $q_{s}$ is the PV anomaly of the vortex patch, i.e.

$$
q_{s}(x, y, t)=\omega_{0} \chi_{\mathscr{D}}(x, y)
$$

where $\mathscr{D}$ specifies the region which the vortex patch occupies at time $t$ and the characteristic function $\chi_{\mathscr{D}}$ equals 1 inside $\mathscr{D}$ and 0 elsewhere. Conventionally, $q_{s}$ and $q_{r}$ are termed the singular and regular parts ( $\psi_{s}$ and $\psi_{r}$ likewise) although here $q_{s}$ is not strictly singular in the sense that its value is bounded throughout the domain. The motion of the vortex boundary $\partial \mathscr{D}$ is described by $\mathrm{d} \boldsymbol{x} / \mathrm{d} t=\boldsymbol{u}(\boldsymbol{x}, t)$ for all $\boldsymbol{x} \equiv(x, y) \in \partial \mathscr{D}$. Hence, from (2.1) and (2.4), we have

$$
\left.q_{r}(x, y)\right|_{t=0}=y
$$

and

$$
\frac{\mathrm{D} q_{r}}{\mathrm{D} t}=0 .
$$

Equation (2.12) shows that the quantity $q_{r}$ is a pointwise conservative field generating the regular flow $\psi_{r}$ in the presence of the planetary PV gradient. This decomposed system (2.10)-(2.12) provides the basis for the implementation of the CASL algorithm. A similar decomposition has been introduced for point vortices (Reznik 1990, 1992) which, however, does not represent the effect of vortex deformation. 


\section{Numerics}

\subsection{The algorithm}

The piecewise-constant nature of the initial condition (2.4) poses difficulties for conventional methods such as finite difference methods (cf. McWilliams \& Flierl 1979) and spectral methods (cf. Sutyrin et al. 1994). In fact, the real difficulty lies not so much with the initial conditions but rather with the inviscid character of the flow. In time, even smooth initial conditions tend to develop practically infinite PV gradients. Conventional methods simply cannot deal with such situations efficiently; significant dissipation must be used to control numerical stability, which implies a continuous erosion of sharp gradients as they try to form (see Mariotti, Legras \& Dritschel 1994 and Yao, Dritschel \& Zabusky 1995). This erosion can have a significant influence on solution accuracy, as demonstrated recently by Dritschel, Polvani \& Mohebalhojeh (1999).

The difficulty of maintaining numerical accuracy when sharp gradients form can be avoided by using contour dynamics (CD, Dritschel 1989), in which dissipation is produced by surgery only and is negligibly small. However, for complex flows, such as considered here, where many auxilliary contours are needed to represent the background planetary vorticity, $C D$ is simply not efficient. Its cost grows with the square of the number of points $n$ used to represent the contours. To improve the efficiency of CD, Dritschel \& Ambaum (1997) combined parts of CD with a standard pseudo-spectral method. In this hybrid algorithm, called the 'contour-advective semiLagrangian' (CASL) algorithm, the slowest aspect of CD - computing the velocity on the contour points - is replaced by a two-step procedure: first, the velocity field is obtained on a regular grid by a conventional spectral approach, and second, this velocity is interpolated onto the contour points. The spectral approach is generally much faster at computing the velocity field, which here amounts only to inverting the Helmholtz operator on the field of PV and taking $x$ and $y$ derivatives of the resulting streamfunction. This requires the PV at grid points, and this PV is obtained using a fast-fill algorithm at $O(n)$ cost. The evolution of the PV field is computed by contour advection, i.e. by solving a system of o.d.e.'s for the contour points, thereby avoiding the CFL stability constraint associated with grid-based advection. The upshot is that the total numerical cost is $O(n)$ plus $O\left(N^{2}\right)$, where $N$ is the grid resolution, compared with $O\left(n^{2}\right)$ in CD. In practice, modest grid resolution can be used while still achieving high overall accuracy.

As mentioned in the previous section, we shall deal directly with the decomposed system (2.10)-(2.12), rather than with the original system (2.1)-(2.5). This amounts to advecting the vortex boundary $\partial \mathscr{D}$ and the isolines of $q_{r}$ by the total velocity field $\boldsymbol{u}=(u, v)$, where

$$
u=-\frac{\partial}{\partial y}\left(\psi_{s}+\psi_{r}\right), \quad v=\frac{\partial}{\partial x}\left(\psi_{s}+\psi_{r}\right)
$$

and where the streamfunctions $\psi_{s}$ and $\psi_{r}$ are found from (2.9).

The flow is simulated within a doubly periodic square domain of size $[-l, l]$, with $l=5 \pi$ here. The coarsest horizontal grid resolution is $\bar{n}_{h}=512$ in each direction, and the PV contour-to-grid conversion is done on a grid twice as fine in each direction $\left(m_{g}=2\right)$. Surgery, or the removal of fine-scale filamentary PV, is applied at the cut-off scale $\delta=1 \times 10^{-3}$ to control the growth of number of contour nodes in prolonged calculations. See Dritschel \& Ambaum (1997) for further details of the CASL algorithm, and Dritschel et al. (1999) for a comparison with other conventional methods. 


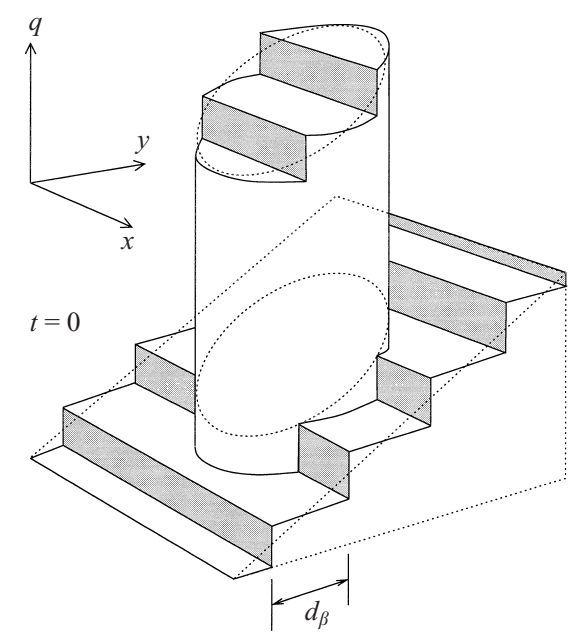

FIGURE 1. An isometric view showing the initial distribution of PV before (dotted) and after (solid) discretization, as given by equation (3.4). The shaded surfaces correspond to the PV discontinuities introduced by discretization, i.e. the $\beta$-contours.

To be compatible with the CASL algorithm, the initial (linear) distribution of $q_{r}$ (2.11) must be discretized into a finite number of steps - see figure 1. The simplest way to do this is to use a certain number, say $n_{\beta}$, of equally spaced steps. These are zonal contours placed at the following positions:

$$
y=\left(k-\frac{1}{2}\right) d_{\beta}-l, \quad k=1, \ldots, n_{\beta},
$$

where

$$
d_{\beta}=\frac{2 l}{n_{\beta}} .
$$

This corresponds to a discretized $q_{r}$ distribution of the form

$$
\left.q_{r}(x, y)\right|_{t=0}=\left[\frac{y}{d_{\beta}}+\frac{1}{2}\right] d_{\beta}
$$

where [.] denotes the integer part. Figure 1 illustrates the initial distribution of total $\mathrm{PV}, q$, before and after discretization. While $q_{r}$ is not $y$-periodic, $q_{r}-y$ is, and this is all that is required to obtain $\psi_{r}$.

The number of $\beta$-contours, $n_{\beta}$, as well as the values of $\bar{n}_{h}, m_{g}$ and $\delta$, limit the accuracy of the numerical simulation. Finite $n_{\beta}$ introduces a source of error which can be kept small if

$$
\frac{l}{n_{\beta} R} \leqslant 1 \quad \text { and } \quad \frac{l}{n_{\beta}\left|\omega_{0}\right|} \ll 1,
$$

or, in terms of the dimensional quantities $l^{*}, R^{*}, \omega_{0}^{*}$ and $\beta$ (see (2.7)), if

$$
\frac{l^{*}}{n_{\beta} R^{*}} \leqslant 1 \quad \text { and } \quad \frac{\beta l^{*}}{n_{\beta}\left|\omega_{0}^{*}\right|} \ll 1 \text {. }
$$

The first criterion guarantees a sufficient resolution of the $\beta$-effect across the vortex, while the second ensures that the PV jump across each contour is small compared to the magnitude of the PV anomaly. In other words, the smaller or weaker the vortex is, the more steps that have to be used. 


\begin{tabular}{ccrccccc}
\hline Vortex & $R$ & $\omega_{0}$ & $n_{\beta}$ & $l / n_{\beta} R$ & $l / n_{\beta}\left|\omega_{0}\right|$ & Figure & Behaviour \\
A & 0.25 & 20 & 150 & 0.42 & $5.2 \times 10^{-3}$ & 2 & weak \\
B & & 80 & & & $1.3 \times 10^{-3}$ & 3 & moderate \\
C & & 240 & & & $4.4 \times 10^{-4}$ & 4 & strong \\
D & 1.0 & 5 & 50 & 0.31 & $6.3 \times 10^{-2}$ & 5 & weak \\
E & & 20 & & & $1.6 \times 10^{-2}$ & 6 & moderate \\
F & & 80 & & & $4.9 \times 10^{-3}$ & 7 & strong
\end{tabular}

TABLE 1. Parameters used in the numerical simulations. The parameters common to all simulations are: $l=5 \pi, \bar{n}_{h}=512, m_{g}=2$ and $\delta=1 \times 10^{-3}$. The classification of the characteristic behaviour is based on observation.

Of course, in principle one would like to choose a very large value of $n_{\beta}$, but in practice the limited computational resources available force a compromise between accuracy and efficiency. In the present work, we have compared simulation results for increasing $n_{\beta}$ until the contour shapes converge to within a desired accuracy. Empirically, it suffices to have $l / n_{\beta} R \sim 1$ and $l / n_{\beta}\left|\omega_{0}\right| \sim 10^{-1}$ for an error tolerance consistent with the prescribed values of $\bar{n}_{h}, m_{g}$ and $\delta$ (see Legras \& Dritschel 1993 for further remarks; see also Dritschel \& Ambaum 1997).

Finally, the finite domain size constrains the duration over which the simulations can reliably approximate the vortex motion in an unbounded domain. This duration is chosen to be the time that it takes the fastest Rossby wave to propagate across the computational domain, or $t=28$ here. Since the maximum wavelength admissible to the square domain is $2 l$, i.e. the length of its edge, the actual non-dimensional maximum Rossby group velocities are $c_{g, \max }=1 /\left(1+(\pi / l)^{2}\right) \approx 0.9615$ westward and $c_{g, \max } / 8$ eastward in the present study where $l=5 \pi$.

\subsection{Numerical results}

Direct numerical calculations have been performed to investigate the long-term behaviour of vortex patches over a range of sizes $R$ and strengths $\omega_{0}$. Only two sets of representative numerical simulations are described here: $R=0.25$ and 1.0, each with three different strengths $\omega_{0}$. A list of the parameters for each simulation is given in table 1, and the corresponding PV evolution is given in figures 2 to 7 . Note that, in these figures, only a fraction of the $\beta$-contours used is shown.

Just as with distributed cyclonic vortices, the vortex patches considered here demonstrate a characteristic northwest migration on the $\beta$-plane, i.e. $\beta$-drift. This migration is accompanied by Rossby wave radiation, particularly in the wake of the vortex. Both of these phenomena are caused by the redistribution of ambient PV. Under the influence of the swirling singular (or vortical) flow $\psi_{s}, \beta$-contours initially begin to wrap around the vortex. The corresponding redistribution of ambient PV leads to the development of a dipolar asymmetry in the regular flow $\psi_{r}$, a pattern termed 'the $\beta$-gyres' (Peng \& Williams 1990; Smith \& Ulrich 1990). These $\beta$-gyres in turn propel the vortex zonally and meridionally. In time, higher modes of asymmetry emerge in $\psi_{r}$. These asymmetries generate Rossby waves in the wake and perturb the boundary of the vortex. This can result in some interesting phenomena if the boundary deformation is unstable (see $\S 5$ below). 

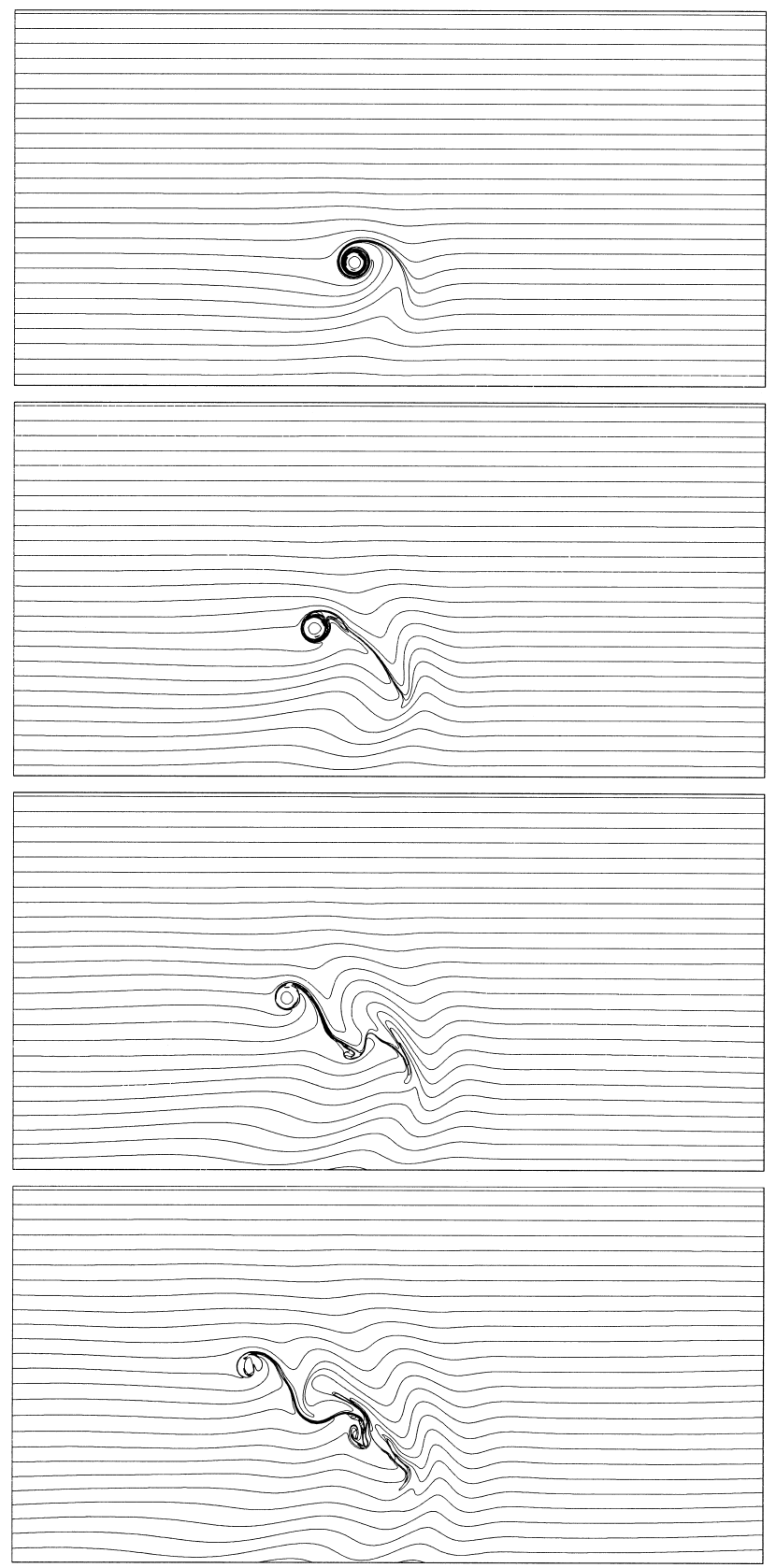

Figure 2. A CASL simulation of the evolution of vortex A $\left(R=0.25\right.$ and $\left.\omega_{0}=20\right)$ on a $\beta$-plane. The frames show the moments $t=7.0,14.0,21.0$ and 28.0, from top to bottom. The domain shown is $[-l, l] \times[-0.25 l, 0.75 l]$, in which only one out of every three $\beta$-contours are shown for brevity.

\section{Beta-drift}

An advantage of the vortex patch model is that the vortex is completely specified by its boundary. That is, all of the vortex properties, such as its position and deformation, can be deduced from the vortex boundary alone. 

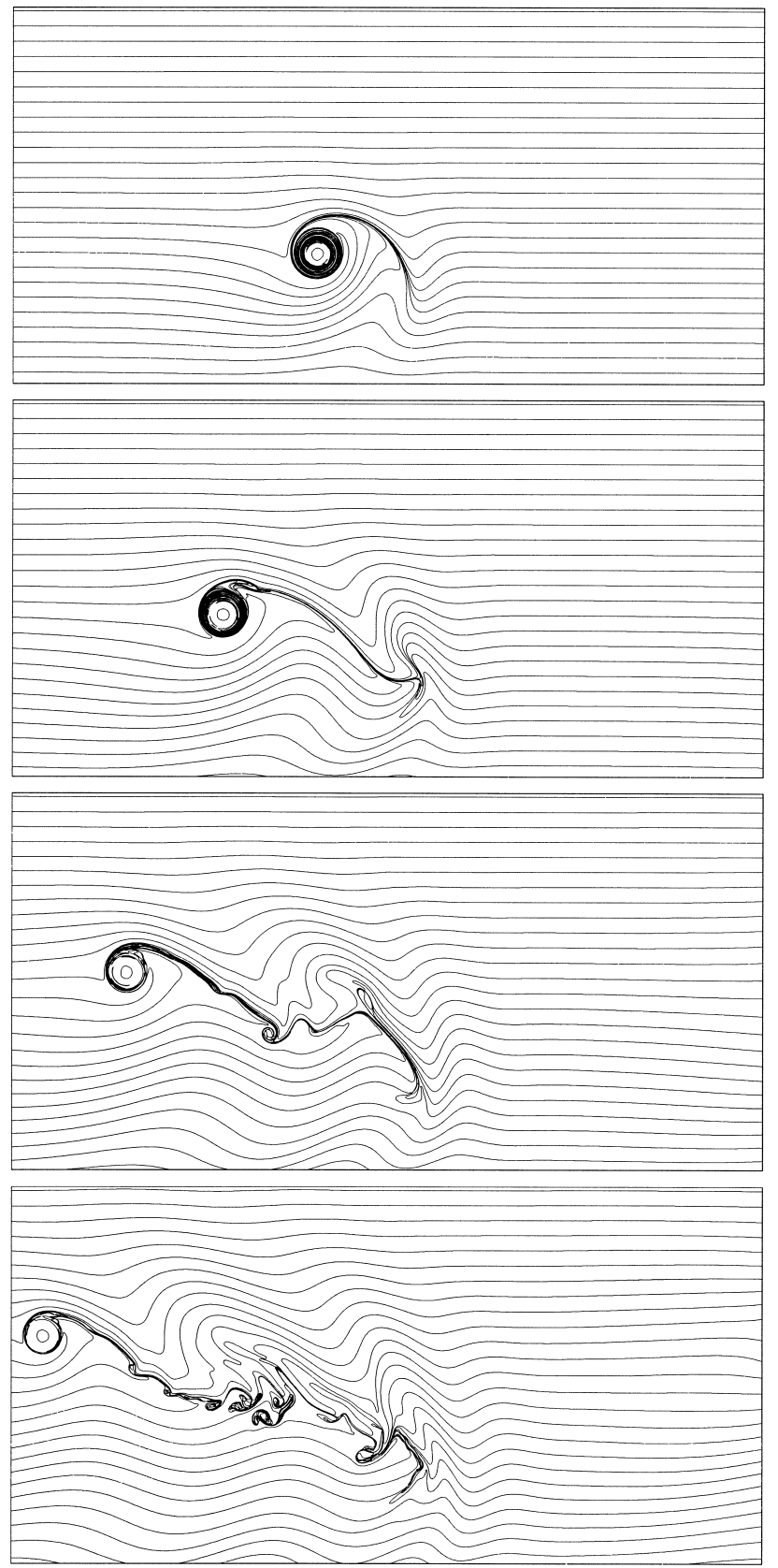

Figure 3. Same as figure 2, except that $\omega_{0}=80$, i.e. vortex B.

The trajectory of a drifting vortex can be described quantitatively given a sensible definition of its centre. Several definitions have been used before, including the vorticity extremum, the streamfunction extremum, and the position of the particle initially at the centre of the vortex (always taken to be circular). The differences between these definitions are discussed by Reznik \& Dewar (1994) and Llewellyn Smith (1997). In the present model (and perhaps in general), it is natural to use the 

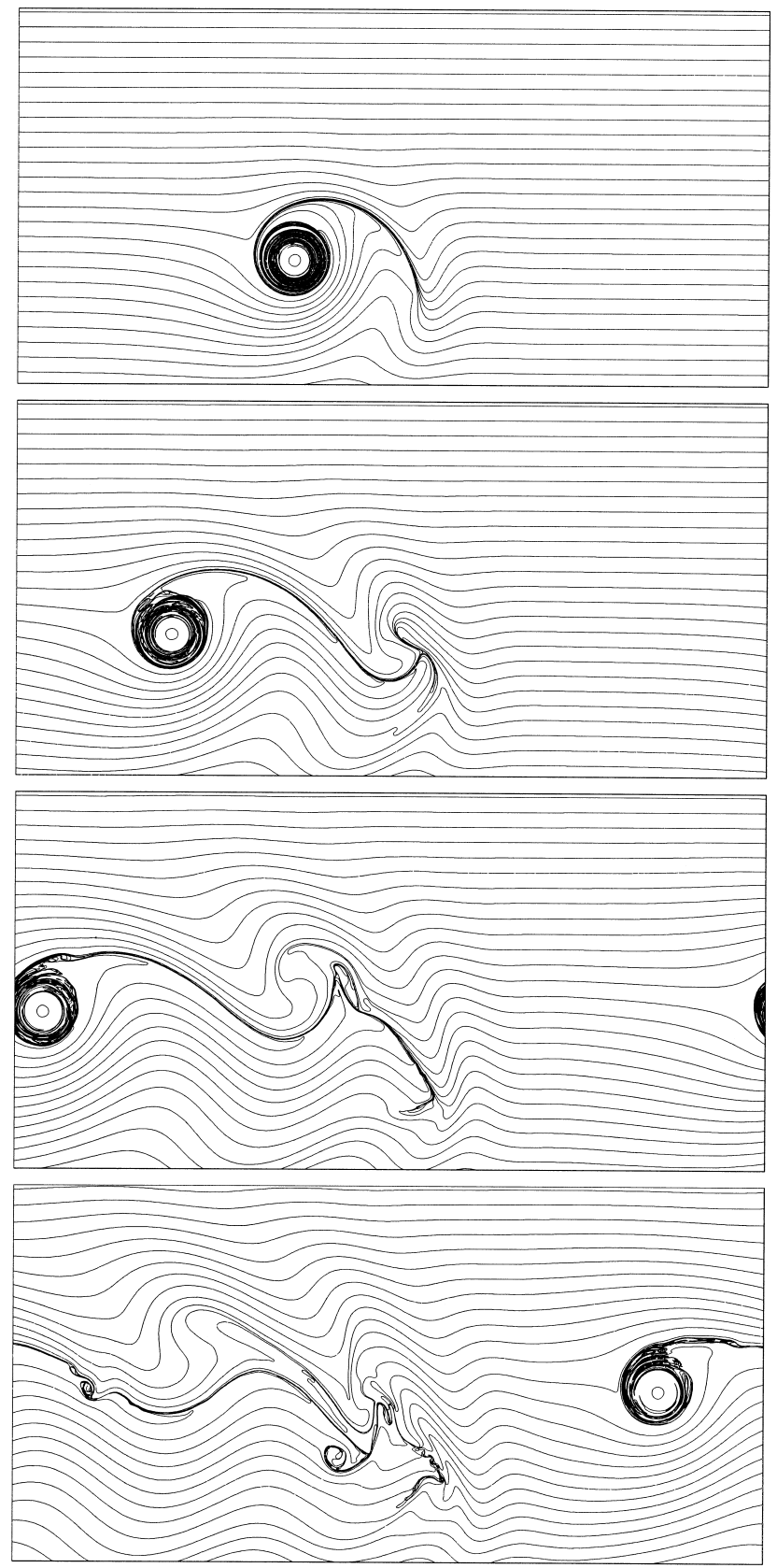

FIgURE 4. Same as figure 2, except that $\omega_{0}=240$, i.e. vortex C.

centroid $\left(x_{0}(t), y_{0}(t)\right)$ as the vortex centre. This choice is natural because it results in vanishing first-order moments in an expansion of the velocity field exterior to the vortex (Dritschel 1993).

In practice, surgery at small scales may cause the vortex patch to fragment, here mainly into filamentary debris surrounding the main vortex. The centre is then calculated from the largest-area contour containing fluid from the original vortex. 

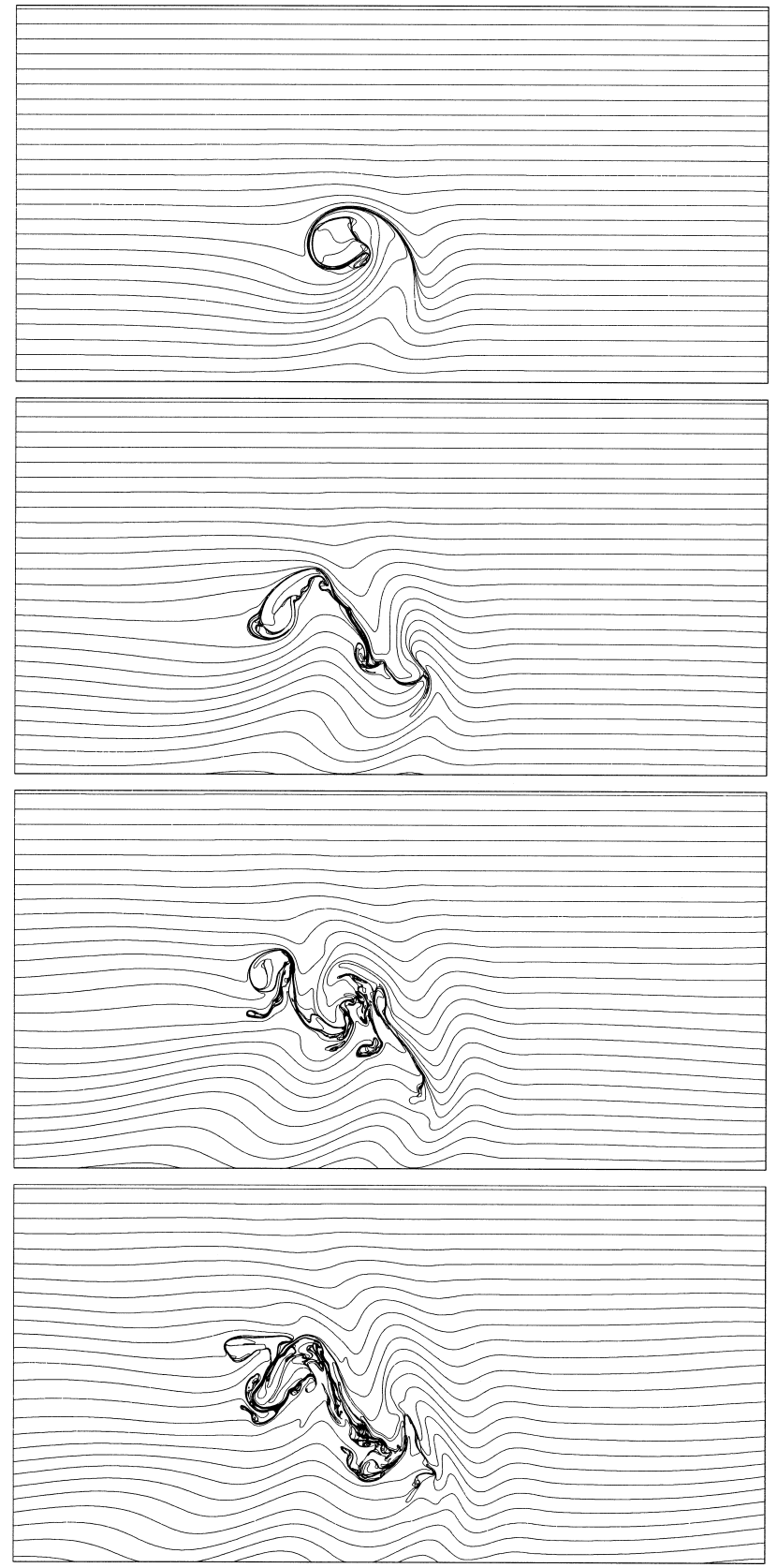

FIgURE 5. Same as figure 2, except that $R=1.0$ and $\omega_{0}=5$, i.e. vortex D. For this bigger vortex patch, all $\beta$-contours are shown.

Note that the boundary deformation, at least before surgery, does not have a direct impact on the motion of the vortex centre. This is because the singular (or self-induced) flow $\psi_{s}$ vanishes at the centroid. The trajectory is thus determined by the accumulated effect of the regular flow $\psi_{r}$ only.

The computed trajectories for $R=0.25$ and 1.0 are shown in figure 8. It is clear that, for both values of $R$, the zonal speed increases with the strength $\omega_{0}$ of the vortex. 

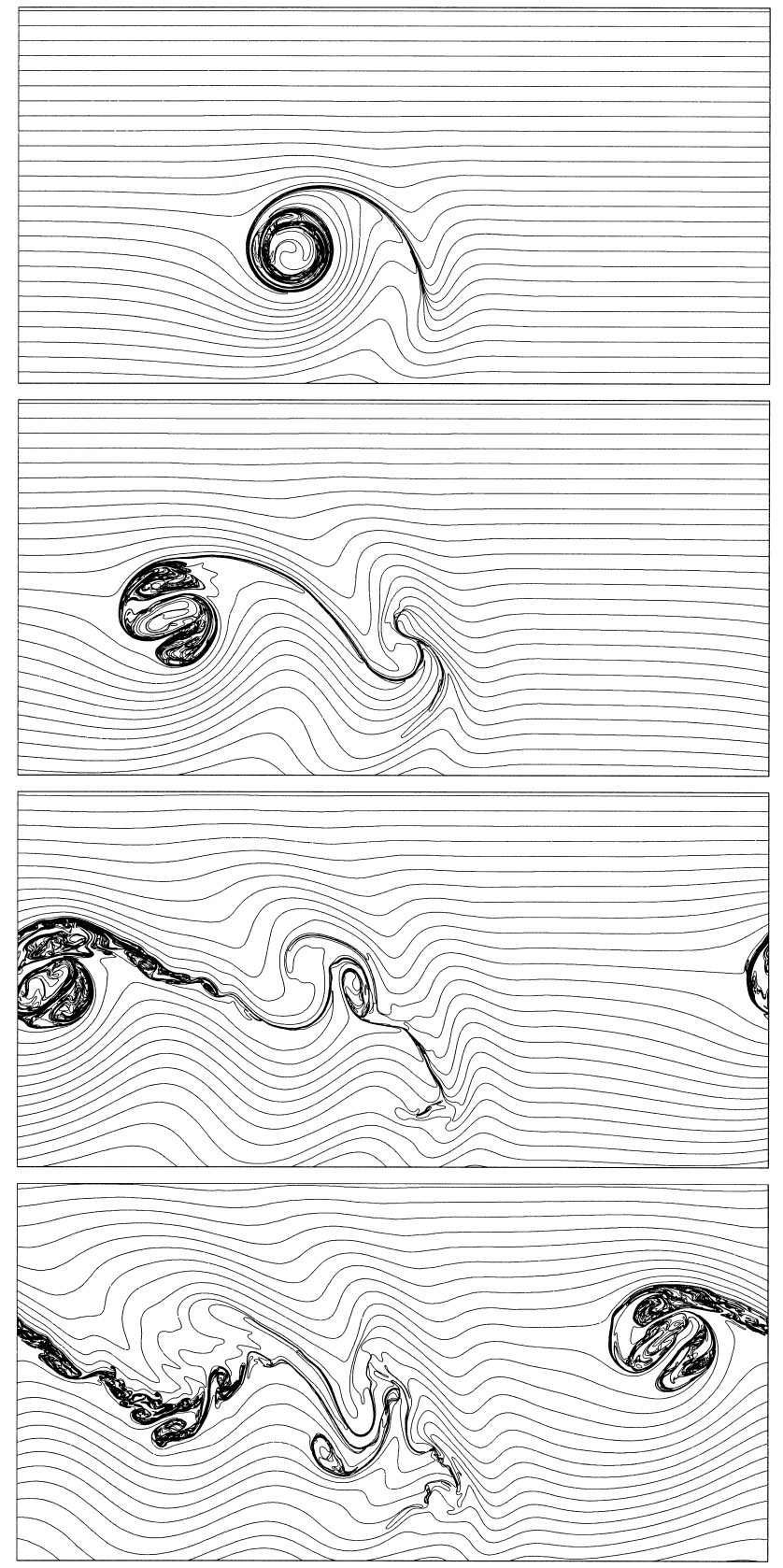

FiguRE 6. Same as figure 5, except that $\omega_{0}=20$, i.e. vortex E.

On the other hand, the meridional speed is not monotonic; vortices of intermediate strength exhibit the largest meridonal speed (see vortices B and E). This remarkable phenomenon was also noted by Rasmussen et al. (1994, figure 2b) and Sutyrin et al. (1994) for Gaussian vortices. The latter authors attributed this phenomenon to the interaction between the vortex and radiating Rossby waves.

The zonal speed appears to be bounded by the maximum westward group speed of Rossby waves $c_{g, \max }$ (see $\S 3$ ), and the meridional speed by the maximum meridional 

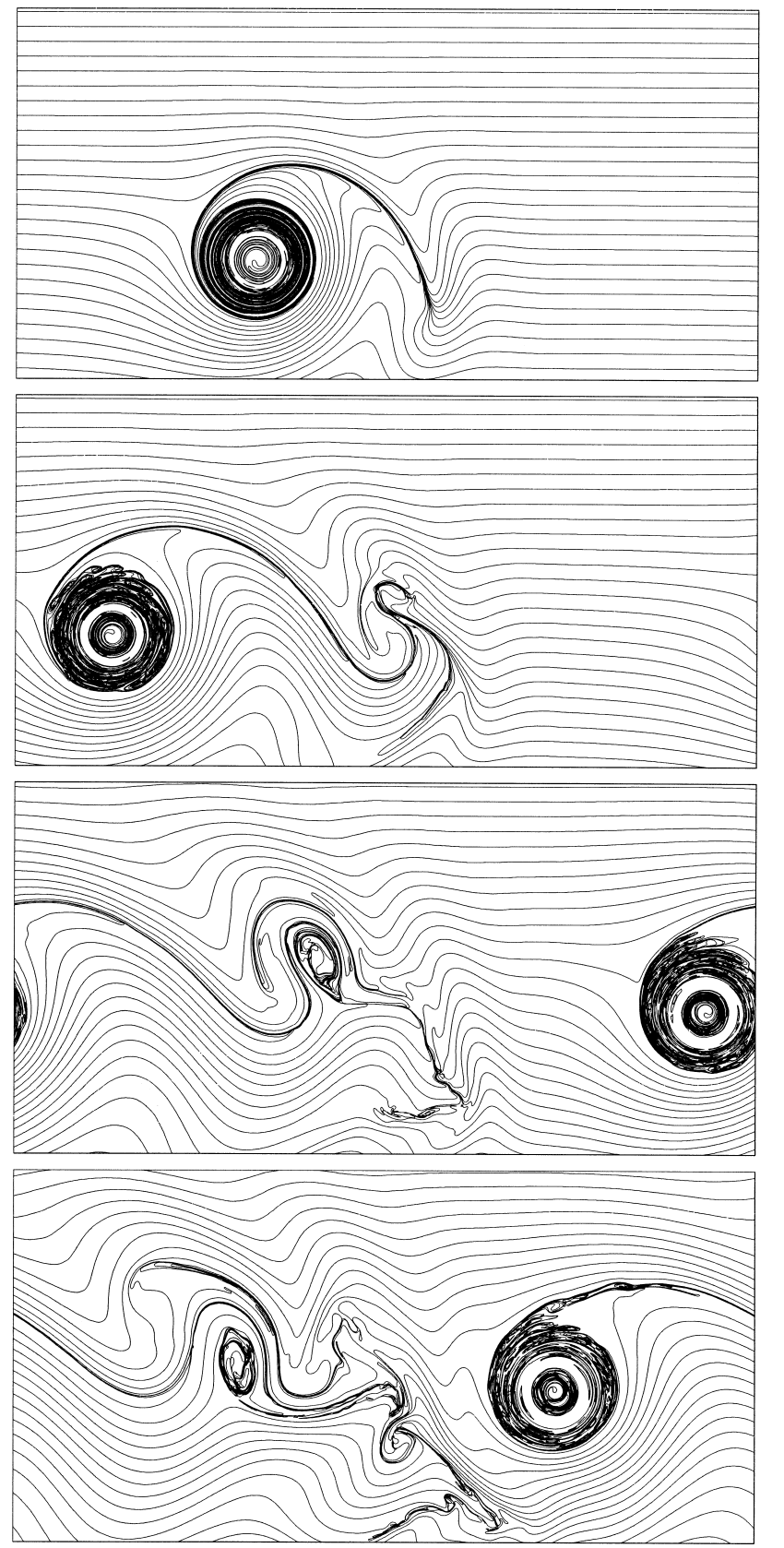

FIGURE 7. Same as figure 5, except that $\omega_{0}=80$, i.e. vortex $\mathrm{F}$.

group speed of Rossby waves, which can be shown to be 0.25 non-dimensional units in our present work (compare with the triangles and diamonds in figure 8). This observation is consistent with the results obtained by McWilliams \& Flierl (1979) and Meid \& Lindemann (1979) who considered Gaussian vortices. Unless they undergo severe deformation (as in the case of vortex D), vortex patches drift at a quasi-steady speed over the time span considered. 

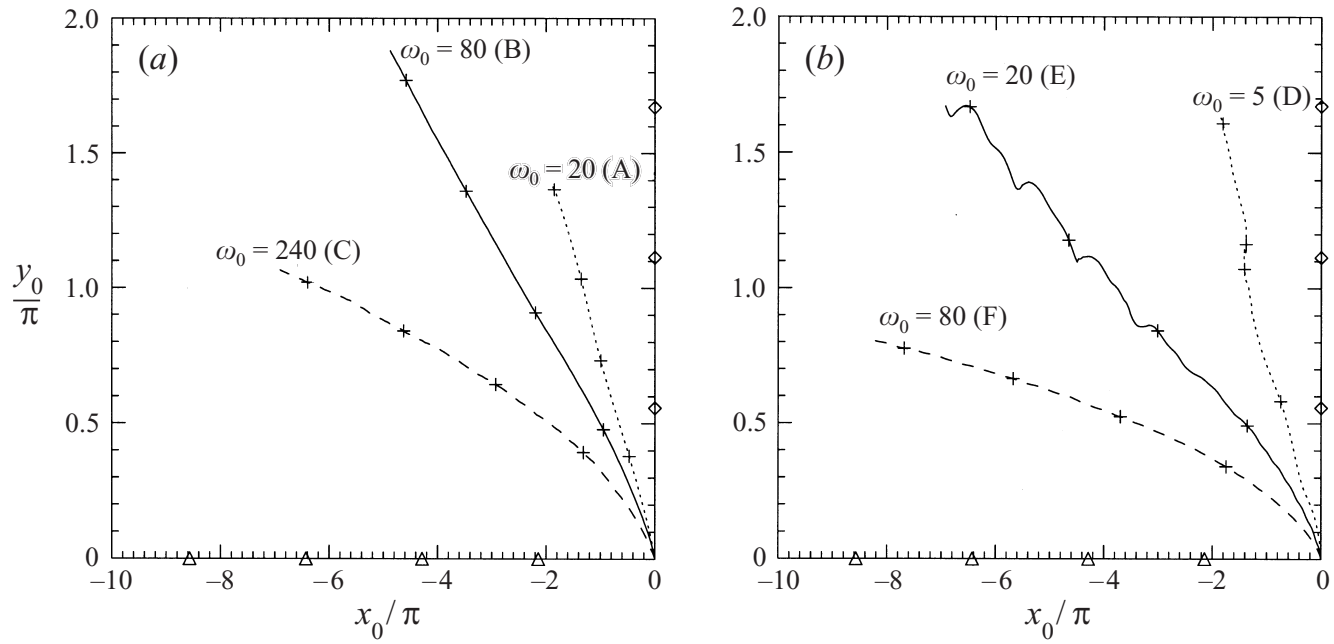

Figure 8. The trajectories of the vortex centre for $(a) R=0.25$ (vortices A to C) and $(b) R=1.0$ (vortices $\mathrm{D}$ to $\mathrm{F}$ ). The crosses $(+)$ specify the instantaneous positions of the vortex centre at time intervals of 7 nondimensional units. The triangles $(\triangle)$ and the diamonds $(\diamond)$ on the axes indicate the corresponding zonal and meridional displacement of a point moving at both maximum zonal $\left(c_{g, \max }\right)$ and maximum meridional $(0.25)$ Rossby group speeds. Note that the fourth diamond is outside the axis frame.

\section{Deformation and instability}

Perturbations to the initially circular vortex boundary develop as soon as the regular flow $\psi_{r}$ is established. Here, the leading-order, elliptical boundary deformation of a vortex is quantified in terms of the aspect ratio $\lambda(\leqslant 1)$ of an analogous ellipse with identical second-order spatial moments (Legras \& Dritschel 1991). The variation of $\lambda$ with time $t$ for our simulations is displayed in figure 9. By comparing parts $(a)$ and $(b)$, one may observe that small vortices $(R=0.25)$ in general remain circular for longer than do large vortices $(R=1.0)$. For each value of $R$, it is also found that the stronger vortices are less vulnerable to deformation. This is logical, as strong compact vortices are less affected by the strain arising from the surrounding Rossby wave field.

Let us now restrict attention to the large vortices first. The weakest such vortex (vortex D) deforms rapidly and is soon torn apart by the straining field present in $\psi_{r}$. The other two stronger vortices (vortices E and F), however, are able to maintain their identity throughout the simulations. An important feature to note for these vortices is the formation of an annulus of fluid which moves along with the vortices. This annulus of fluid is called the trapped zone (cf. Korotaev \& Fedotov 1994). It consists of relative vorticity of the opposite sign acquired as the vortex displaces meridionally (a consequence of the conservation of PV). As a result, the vortex becomes shielded, i.e. its net circulation (including the annulus) tends to zero in time.

At this stage of evolution, the vortex evolves in a quasi-steady way. In time, the vortex may deform into an ellipse and may suddenly destabilize, fragmenting into smaller structures. This behaviour, as shown below, can be understood by considering the linear stability of the vortex in the absence of the $\beta$-effect, following an earlier analysis by Flierl (1988).

To set the stage, we first need to define the trapped-zone radius $\tilde{R}$ (the outer boundary of the annulus). A polar coordinate system $(r, \theta)$ is fixed at the vortex 

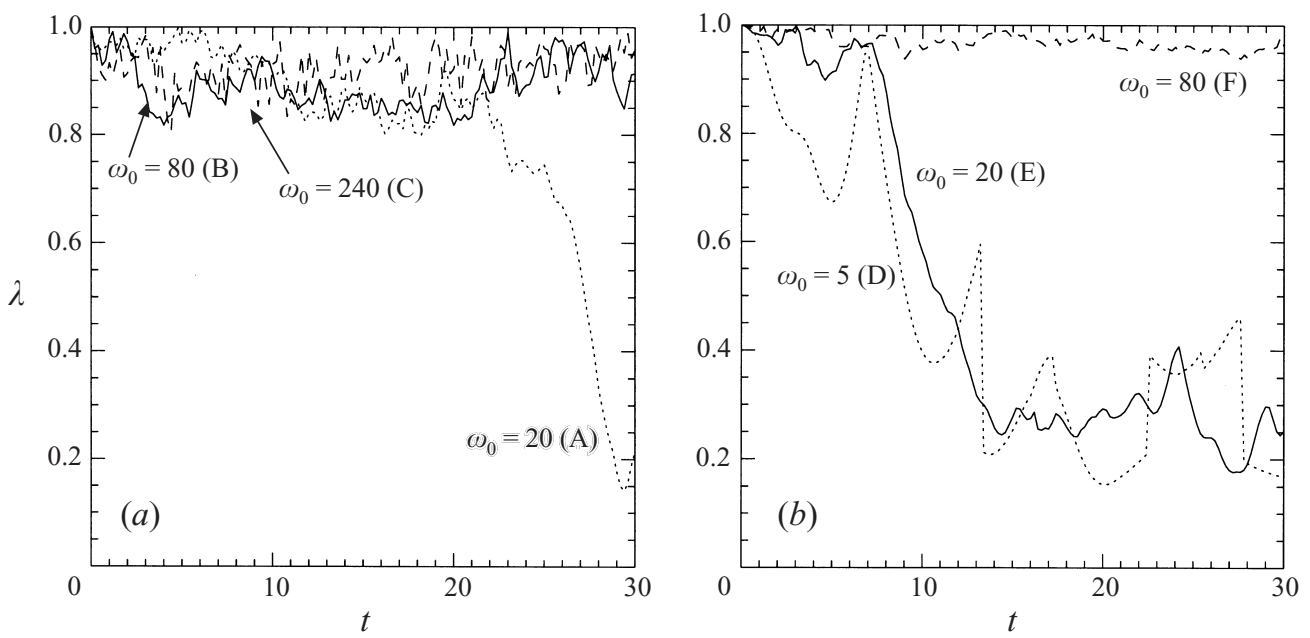

FIGURE 9. The temporal variation of the aspect ratio $\lambda$ of the vortex boundary for $(a) R=0.25$ (vortices A to $\mathrm{C}$ ) and (b) $R=1.0$ (vortices $\mathrm{D}$ to $\mathrm{F}$ ).

centre such that

$$
\boldsymbol{x}-\boldsymbol{x}_{0}=(r \cos \theta, r \sin \theta)
$$

where $\boldsymbol{x}=(x, y)$ and $\boldsymbol{x}_{0}=\left(x_{0}, y_{0}\right)$. The regular part $q_{r}(t, r, \theta)$ is expanded as follows:

$$
q_{r}-y=q_{0}(t, r)+\sum_{n=1}^{\infty}\left(q_{n}^{c} \cos n \theta+q_{n}^{s} \sin n \theta\right) .
$$

The structure of the first few modes $\left(q_{0}, q_{1}^{c}\right.$ and $\left.q_{1}^{s}\right)$ for vortices $\mathrm{B}$ and $\mathrm{C}$ is given in figure 10. Note the steep jump in $q_{0}$ at the time shown. This is anticipated to be a general feature as discussed in the next section. Between the steep jump and the origin, $q_{0}$ resembles a flat-bottomed well, where the PV has been homogenized by the rapid swirling flow in $\psi_{s}$. It is reasonable, therefore, to identify the position of this steep jump as the trapped-zone radius $\tilde{R}$.

In practice, we define $\tilde{R}$ to be the radius from the vortex centre to the mid-point of two consecutive radial nodes $r_{1}<r_{2}$, separated by a distance equal to the size of the fine underlying grid, where

$$
q_{0}\left(r_{2}\right)-q_{0}\left(r_{1}\right)
$$

is maximum. (If such a mid-point is not unique, we choose the one furthest from the vortex centre. This is sufficient because $q_{0}$ is generally smooth outside the trapped zone.) The average relative vorticity acquired inside the trapped zone is approximated by minus $y_{0}$ (this is an excellent approximation, as can be seen by comparing the flat-bottom value of $q_{0}$ in figure 10 with the corresponding position of the vortex centre in figure $8 a$ ).

The Appendix describes the derivation of the linear stability criterion for various modes of boundary perturbation. Basically it repeats the calculation of Flierl (1988), except that Flierl considered a barotropic basic flow $\left(R_{d}=\infty\right)$. In our present work, both the basic flow and the disturbances are equivalent barotropic $\left(R_{d}\right.$ is finite). Figure 11 shows the part of the $\left(y_{0} / \omega_{0}, \tilde{R} / R\right)$-plane which is unstable to $m=2$ (elliptical) and $m=3$ modes along with the results obtained by direct numerical simulation (cf. figure 6 of Flierl 1988). Figure 11(a) shows the case $R=0.25, \omega_{0}=20$ 

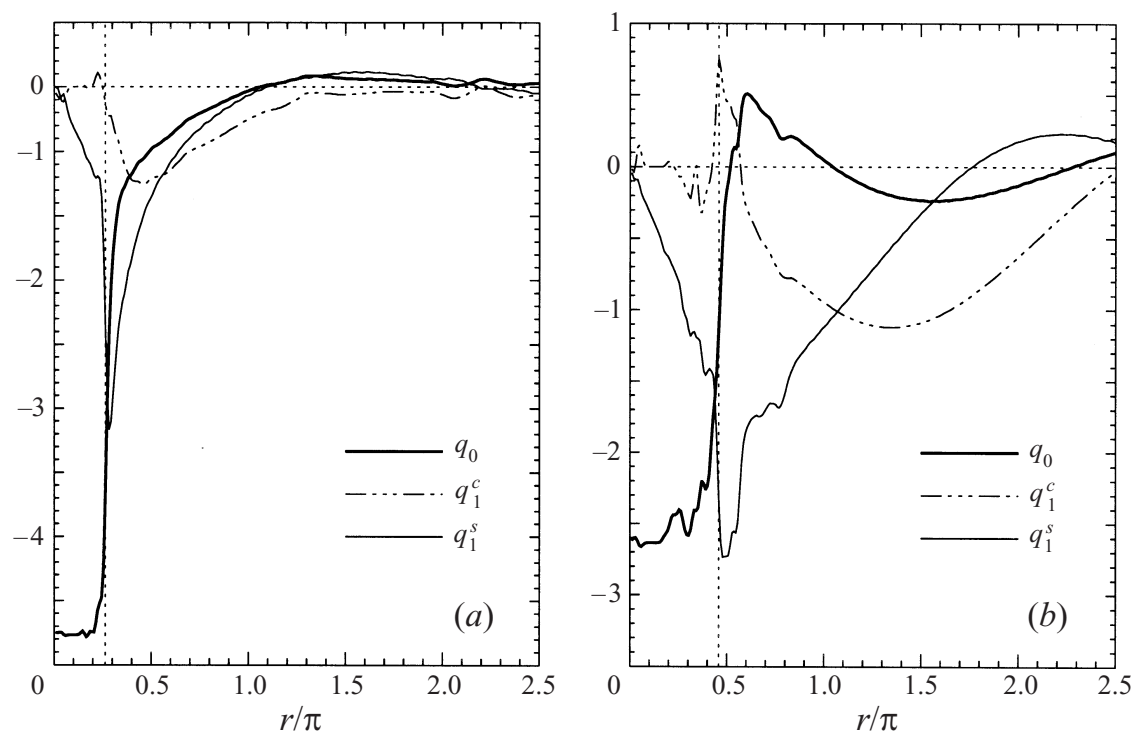

FiguRE 10. The Fourier decomposition (see (5.2)) of the regular part $q_{r}$ for $(a)$ vortex B and $(b)$ vortex $\mathrm{C}$ at time $t=21.0$. The vertical dotted line indicates the trapped-zone radius $\tilde{R}$ which is defined to be the radial distance from the vortex centre with the maximum gradient of $q_{0}$.
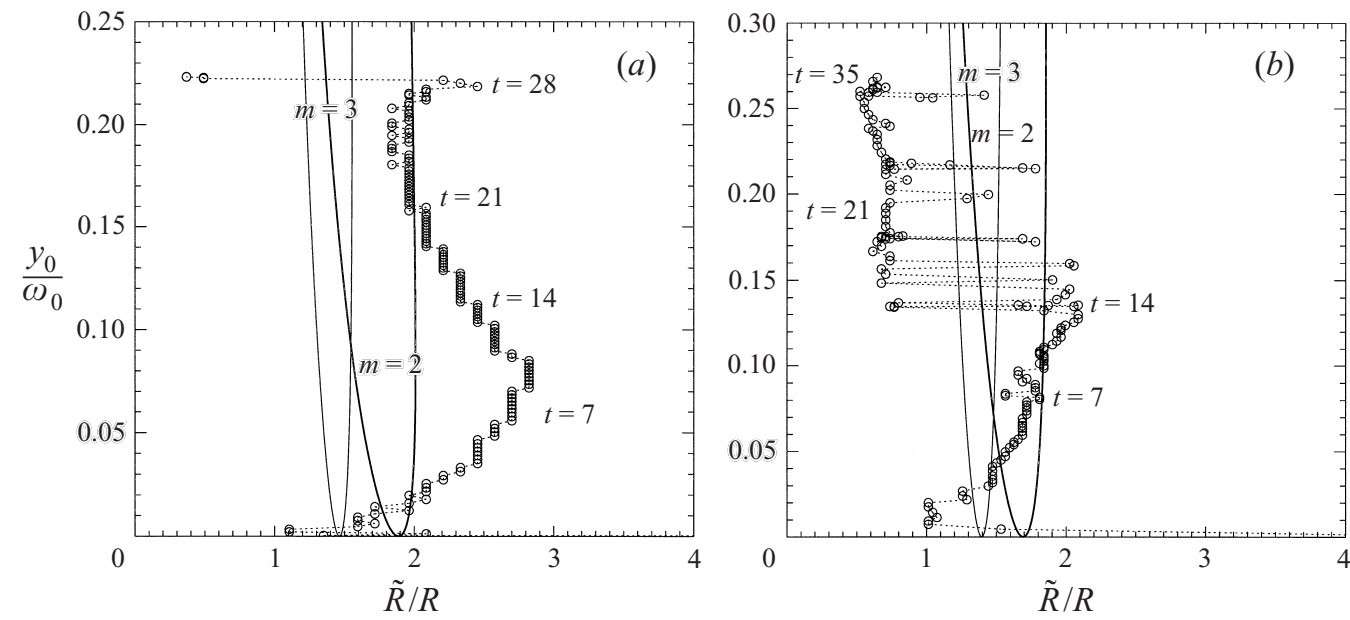

FIGURE 11. Stability curves for $m=2$ (bold) and $m=3$ (thin) wavenumbers for $(a)$ vortex A and (b) vortex E. The regions enclosed by these curves are unstable. Circles (o) joined by dotted lines are obtained from the numerical simulations.

(vortex A) while $11(b)$ shows the case $R=1.0, \omega_{0}=20$ (vortex E). It should be noted that the value of $\tilde{R}$ calculated by our method is inaccurate at the beginning of the simulation when the trapped zone has not been well established. This method also breaks down when the vortex suffers severe boundary deformation. This is the reason for the abrupt decrease and subsequent fluctuation in the value of $\tilde{R} / R$ around $y_{0} / \omega_{0} \approx 0.13$ in figure $11(b)$. This sharp decrease, in fact, corresponds to the moment when the tripolar vortex emerges in case E. Generally, an initial increase in $\tilde{R}$, followed by a gradual decrease, is observed for both small and large vortices. 


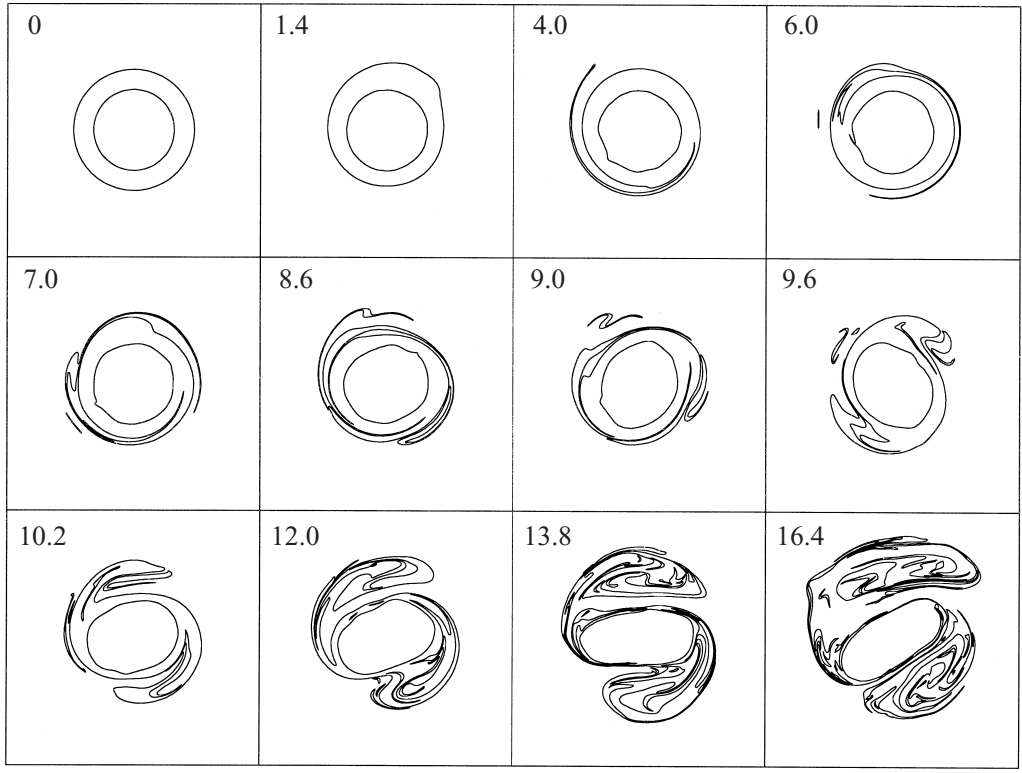

FIGURE 12. The detailed development of the rotating tripolar structure (first shown in figure 6) is revealed with the help of an initially circular passive tracer contour of radius $r=1.5$ (outer contour). The inner contour is the vortex boundary.

Figure 11(b) shows that vortex E has traversed a large part of the unstable region for the elliptical $(m=2)$ mode. This vortex severely deforms and a rotating tripolar structure emerges, a manifestation of the mode-2 instability (cf. Carton \& Legras 1994). This phenomenon was also observed for isolated Gaussian vortices moving on a $\beta$-plane, as noted by Sutyrin et al. (1994). The detailed development of the tripolar structure is displayed in figure 12 with the help of an outer passive tracer contour. The tripolar rotation and the elliptical vortex core together cause the vortex to wobble, as seen in the wavy shape of its trajectory in figure 8. By contrast, the small vortex A (of the same strength) in figure 11(a) falls into the unstable region for $m=2$ only in its late stage of evolution. Figure 2 shows that this vortex remains stable until $t=28$ (last frame shown), when a small tripolar structure emerges.

It should be remarked that the actual evolution of the vortex is intrinsically nonlinear. A slight discrepancy is therefore expected between the actual onset of instability and that predicted by linear theory. Moreover, that theory neglects $\beta$.

\section{Formation of steep PV gradients}

As shown in figure 10, a steep PV gradient in $q_{0}$ exists near the periphery of the trapped zone. This trapped zone is formed by the entrainment of fluid from the vicinity of the drifting vortex. It moves along with the vortex and forces the external iso-PV contours ( $\beta$-contours) out of its way. Since such contours are material curves, they cannot be crossed by any of the fluid particles advected along with the vortex. Thus, as the vortex approaches, these contours embrace the trapped zone and are subsequently dragged along with it (but not trapped by it). The repeated engulfment of the trapped zone by these external iso-PV contours results in the development of a steep PV gradient.

Another steep PV gradient develops in the wake of the vortex, and is called 
$t=0$

○

$$
t=7.0
$$
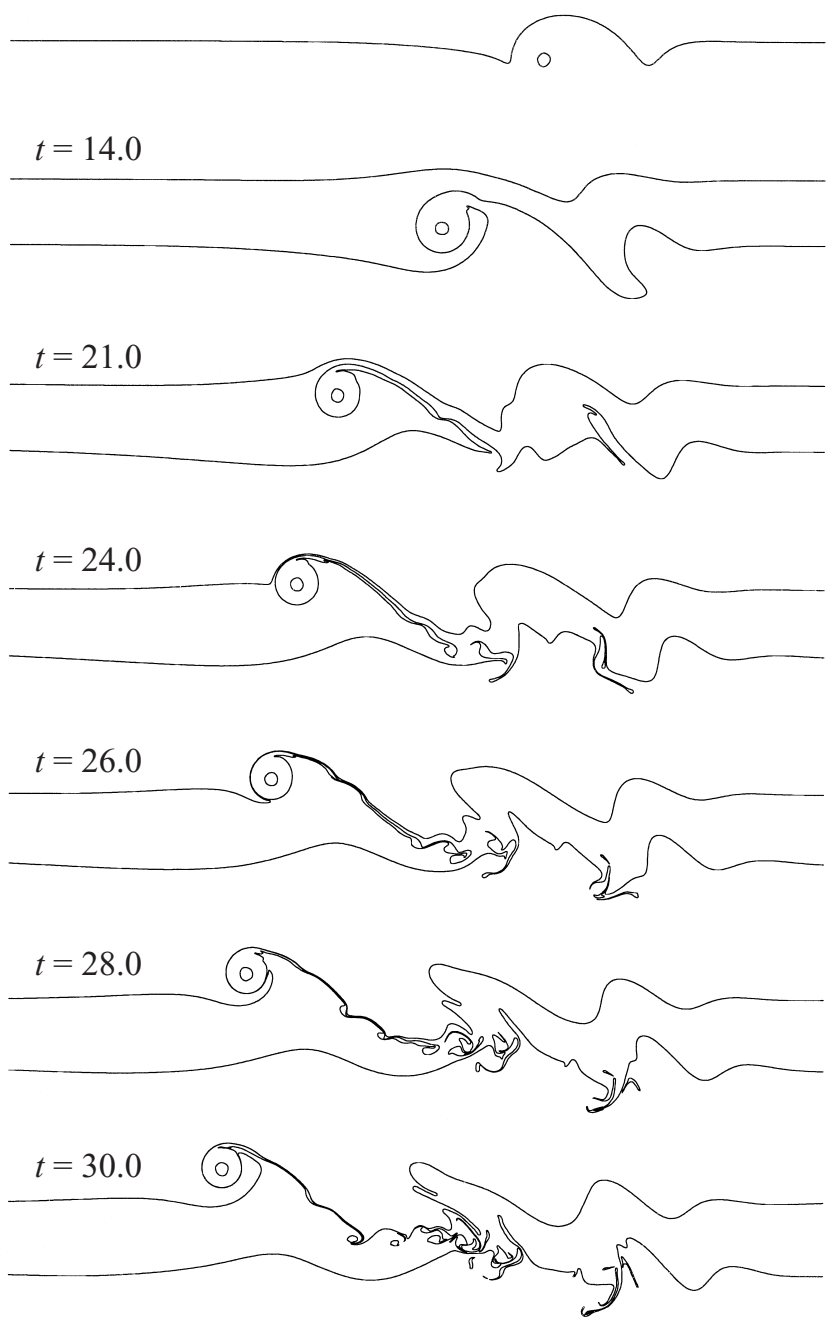

FiguRE 13. The formation of the trailing front and the sharp PV gradient around the trapped zone is illustrated by the evolution of two selected $\beta$-contours, namely $y=0.42 \pi$ and $1.5 \pi$, for the vortex $\mathrm{B}\left(R=0.25\right.$ and $\left.\omega_{0}=80\right)$. The closed contour is the vortex boundary. The domain is shifted by $0.5 \pi$ eastwards. At $t=21$ and 24 , we see that these two $\beta$-contours, being pushed northwards by the vortex, approach each other. Parts of these contours become coincident and indistinguishable subsequently, indicating the presence of very large PV gradients there.

the 'trailing front'. It is formed by the same mechanism as described above. This front grows in length as the vortex propagates. The trailing front provides the only communication (of fluid particles) between the trapped zone and the rest of the domain. In fact, the trapped zone leaks, and it leaks directly into the ribbon of fluid along the trailing front. 
The formation of these steep PV gradients is vividly depicted in figure 13, which shows the detailed evolution of selected $\beta$-contours for vortex $\mathrm{B}$. It is remarked that the mechanism described above is applicable to distributed vortices as well. Steep PV gradients are anticipated to form in any inviscid evolution of an isolated vortex on the $\beta$-plane.

\section{Conclusions}

The long-term inviscid evolution of an initially circular vortex patch on a $\beta$-plane has been examined at unprecedented resolution using the CASL algorithm. The successful application of this algorithm demonstrates its effectiveness and robustness for simulating geophysical flows having a non-uniform ambient PV distribution. Two criteria have been developed to ensure accurate flow simulations. The first is that the spacing between ambient PV contours must be small compared to the vortex diameter. The second is that the PV jump across each ambient PV contour must be small compared to the vortex PV anomaly. These criteria, summarized in (3.5) or (3.6), serve as a fundamental guideline for discretizing any initial continuous PV profile.

The flow decomposition described in this work allows us to split the whole flow field into two parts. The regular part corresponds to the flow induced by the redistribution of ambient PV. The singular part corresponds to the flow generated by the vortex. The singular flow is solely determined by the vortex, or even just its boundary for a vortex patch. This decomposition turns out to be natural for the implementation of the CASL algorithm. It also appears to be useful for the analysis of the vortex motion and deformation.

In our analysis, we have used the centroid as the vortex centre, in terms of which we have obtained the trajectories of the vortices as a function of their size and strength. We have found that the zonal speed of a vortex increases with its strength. However, the maximum (average) meridional speed occurs for vortices of intermediate strength (for the time span considered in our simulations). The latter conclusion was also reached in previous studies of distributed (Gaussian) vortices.

Unlike previous studies, we have considered the effect of vortex size. Large vortex patches have been found to deform more readily than small vortices. Moreover, vortices acquire an annular region of trapped fluid (called the trapped zone) which cancels the circulation of the vortex core. This trapped zone shrinks in size but becomes more intense while the vortex displaces meridionally. Following and extending the results of Flierl (1988), a linear stability analysis for shielded vortices has been performed for $\beta=0$. Despite not including the ambient PV gradient explicitly, this analysis is nevertheless capable of explaining the emergence of tripolar structures in our simulations via the destabilization of an elliptical boundary perturbation.

Apart from the slight dissipation due to fine-scale surgery, the CASL algorithm is essentially inviscid. It has enabled us to capture the development of abrupt PV gradients around the trapped zone and in the trailing front making up the wake of the vortex. These virtual PV discontinuities are formed by the wrapping of external iso-PV contours ( $\beta$-contours) around the trapped zone as the vortex tries to propagate through them. Without viscosity, these sharp gradients persist and the background planetary vorticity is permanently modified. Since this mechanism is also applicable to distributed vortices, steep PV gradients are expected to be a general feature of vortex motion on the $\beta$-plane.

It is also worth mentioning that the observed disruption of the initial planetary 


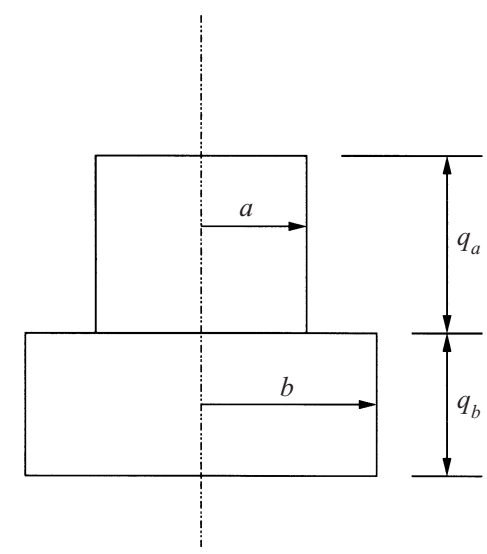

FIGURE 14. Cross-section of the two-level circular vortex patch, approximating the local PV field observed in our numerical simulations.

vorticity gradient undermines the use of such smooth gradients in conjunction with strong vortices. If no mechanism, such as viscosity, is available to restore a smooth gradient, the effect of many vortex passages through a region of varying planetary vorticity must be to focus the gradients into narrow regions, i.e. into jets. This may explain the predominance of such jets in the oceans, the atmosphere and other planetary atmospheres.

Finally, it is remarked that a quasi-steady drift speed has been observed for sufficiently strong vortices, shortly after the simulation commences. Koroteav \& Fedotov (1994) gave approximate analytical formulae for the zonal and meridional drift speed of an isolated Gaussian vortex (see their equations (4.30) and (4.31)). They expressed these speeds in terms of the slow-varying meridional displacement of the vortex. In their derivation, the domain is divided into two parts by the separatrix of the streamfunction. This separatrix is assumed to be the boundary of the trapped zone. The corresponding inner and outer solutions are then matched along this separatrix or the trapped-zone boundary. This domain decomposition technique is in fact natural to our present problem. As figure 10 in $\S 5$ suggests, the flow inside and outside the trapped zone is quite different. An attempt has been made by Lam (1998) to derive corresponding analytical formulae for an initially circular vortex patch. The domain is divided in the same way. A steady solution corresponding to a dipole with a zonal axis is obtained for the inner solution for $r<\tilde{R}$ (cf. equation (7.1) of Sutyrin \& Flierl (1994), in which $r_{M}$ stands for the radius of the outermost contour, instead of the trapped-zone radius). The outer solution is 'solved' by a heuristic argument. Although the inner solution shows a good agreement with numerical calculations, the outer solution is not accurate enough. Efforts are being made to establish a better outer solution. Such predictions of the vortex drift speed are important and worth further research, particularly in the more general context where the ambient PV gradient is non-uniform (e.g. over topographic features in the ocean). They may not only allow us to have a better quantitative estimate of the impact (due to passive tracer transport) of geophysical vortices on their surrounding environment, but may also enhance our understanding of the role played by Rossby wave radiation in the propagation of vortices.

J. S.-L. L. acknowledges support from the Cambridge Commonwealth Trust through the Prince Philip Graduate Exhibition, and is grateful to P.F. Linden for suggesting 
this interesting problem. D.G.D. was supported by a fellowship from the Natural Environment Research Council. The computations were performed on the Hitachi S3600 made available by courtesy of the High Performance Computing Facilities at the University of Cambridge and the High Performance Computing Centre at Maidenhead, UK. The authors would like to thank the anonymous referees for valuable comments and suggesting helpful references.

\section{Appendix}

The linear stability criterion (corresponding to equation (3.5) of Flierl 1988) for a circular vortex consisting of two uniform regions of PV (cf. figure 14) on an $f$-plane can be shown to be

$$
\begin{aligned}
{\left[\frac{\bar{V}(a)}{a}-q_{a} I_{m}\left(\frac{a}{R_{d}}\right) K_{m}\left(\frac{a}{R_{d}}\right)-\frac{\bar{V}(b)}{b}\right.} & \left.+q_{b} I_{m}\left(\frac{b}{R_{d}}\right) K_{m}\left(\frac{b}{R_{d}}\right)\right]^{2} \\
& <-4 q_{a} q_{b} I_{m}^{2}\left(\frac{a}{R_{d}}\right) K_{m}^{2}\left(\frac{b}{R_{d}}\right)
\end{aligned}
$$

where $a$ and $q_{a}$ are the radius of and the PV jump across the inner contour of the vortex. Likewise $b$ and $q_{b}$ are defined for the outer contour. The integer $m$ denotes the azimuthal wavenumber of the boundary perturbation, taken to be proportional to $\exp (\mathrm{im}(\theta-\sigma t))$ on each contour, where $\sigma$ denotes the angular velocity at which the perturbation propagates along both contours of the vortex. The azimuthal velocity $\bar{V}(r)$ generated by the unperturbed vortex is determined by

$$
\left[\frac{\mathrm{d}^{2}}{\mathrm{~d} r^{2}}+\frac{1}{r} \frac{\mathrm{d}}{\mathrm{d} r}-\left(\frac{1}{R_{d}^{2}}+\frac{1}{r^{2}}\right)\right] \bar{V}(r)=-q_{a} \delta(a-r)-q_{b} \delta(b-r)
$$

where $\delta$ is the delta function. This implies that

$$
\begin{gathered}
\frac{\bar{V}(a)}{a}=\frac{b}{a} q_{b} K_{1}\left(\frac{b}{R_{d}}\right) I_{1}\left(\frac{a}{R_{d}}\right)+q_{a} K_{1}\left(\frac{a}{R_{d}}\right) I_{1}\left(\frac{a}{R_{d}}\right), \\
\frac{\bar{V}(b)}{b}=\frac{a}{b} q_{a} K_{1}\left(\frac{b}{R_{d}}\right) I_{1}\left(\frac{a}{R_{d}}\right)+q_{b} K_{1}\left(\frac{b}{R_{d}}\right) I_{1}\left(\frac{b}{R_{d}}\right) .
\end{gathered}
$$

Equations (A 3) and (A 4) are the crucial difference between our present treatment and that of Flierl (1988). The latter studied a barotropic vortex subject to baroclinic perturbations and, therefore, the angular velocity along the contours of the vortex used there is a special case of (A 3) and (A 4) with $R_{d} \rightarrow \infty$.

For our present problem, we have

$$
R_{d}=1, \quad a=R, \quad q_{a}=\omega_{0}, \quad b=\tilde{R}, \quad q_{b}=-y_{0},
$$

in terms of which the stability criterion (A 1) becomes

$$
\left[\frac{\bar{V}(R)}{R}-\omega_{0} I_{m}(R) K_{m}(R)-\frac{\bar{V}(\tilde{R})}{\tilde{R}}-y_{0} I_{m}(\tilde{R}) K_{m}(\tilde{R})\right]^{2}<4 \omega_{0} y_{0} I_{m}^{2}(R) K_{m}^{2}(\tilde{R})
$$

and the angular velocities at the PV discontinuities are

$$
\frac{\bar{V}(R)}{R}=\left[\omega_{0} K_{1}(R)-\frac{\tilde{R}}{R} y_{0} K_{1}(\tilde{R})\right] I_{1}(R)
$$




$$
\frac{\bar{V}(\tilde{R})}{\tilde{R}}=\left[\frac{R}{\tilde{R}} \omega_{0} I_{1}(R)-y_{0} I_{1}(\tilde{R})\right] K_{1}(\tilde{R}) .
$$

For any particular choice of parameters $\left(R, \omega_{0}\right)$, we can easily verify that (A 6$)$ is a quadratic inequality in $y_{0} / \omega_{0}$ for any given $\tilde{R} / R$. Therefore, the stability curve has at most two solutions for $y_{0} / \omega_{0}$ for each value of $\tilde{R} / R$. Figure 11 shows the stability curves for wavenumbers $m=2$ and $m=3$ for vortices A and E.

\section{REFERENCES}

ADEM, J. 1956 A series solution for the barotropic vorticity equation and its application in the study of atmospheric vortices. Tellus VIII, 346-372.

Carnevale, C. F., Kloosterziel, R. C. \& Heijst, G. J. F. van 1991 Propagation of barotropic vortices over topography in rotating tank. J. Fluid Mech. 223, 119-139.

Carton, X. \& Legras, B. 1994 The life-cycle of tripoles in two-dimensional incompressible flows. J. Fluid Mech. 267, 53-82.

DRITSCHEL, D. G. 1989 Contour dynamics and contour surgery: numerical algorithms for extended, high-resolution modelling of vortex dynamics in two-dimensional, inviscid, incompressible flows. Comput. Phys. Rep. 10, 77-146.

Dritschel, D. G. 1993 A fast contour dynamics method for many-vortex calculations in twodimensional flows. Phys. Fluids A 5, 173-186.

Dritschel, D. G. \& Ambaum, M. H. P. 1997 A contour-advective semi-Lagrangian numerical algorithm for simulating fine-scale conservative dynamical fields. Q. J. R. Met. Soc. 123, 1097 1130 .

Dritschel, D. G., Polvani, L. M. \& Mohebalhojeh, A. R. 1999 The contour-advective semiLagrangian algorithm for the shallow-water equations. Mon. Wea. Rev. 127, 1151-1165.

Firing, E. \& BeARDSley, R. C. 1976 The behaviour of a barotropic eddy on a $\beta$-plane. J. Phys. Oceanogr. 6, 57-65.

FLIERL, G. R. 1988 On the instability of geostrophic vortices. J. Fluid Mech. 197, 349-388.

Fuglister, F. \& Worthington, V. 1951 Some results of a multiple ship survey of the Gulf Stream. Tellus 3, 1-14.

Hoskins, B. J., McIntyre, M. E. \& Robertson, A. W. 1985 On the use and significance of isentropic potential-vorticity maps. Q. J. R. Met. Soc. 111, 877-946.

Korotaev, G. R. \& Fedotov, A. R. 1994 Dynamics of an isolated barotropic eddy on a beta-plane. J. Fluid Mech. 264, 277-301.

LAM, J. S. L. 1998 The genesis, motion and stability of a vortex in a planetary vorticity gradient. PhD Dissertation, University of Cambridge, $152 \mathrm{pp}$

Legras, B. \& Dritschel, D. G. 1991 The elliptical model of two-dimensional vortex dynamics. Part I: the basic state. Phys. Fluids A 3, 845-854.

Legras, B. \& Dritschel, D. G. 1993 A comparison of the contour surgery and pseudo-spectral methods. J. Comput. Phys. 104, 287-301.

Llewellyn Smith, S. G. 1997 The motion of a non-isolated vortex on the beta-plane. J. Fluid Mech. 346, 149-179.

Mariotti, A., Legras, B. \& Dritschel, D. G. 1994 Vortex stripping and the erosion of coherent structures in two-dimensional flows. Phys. Fluids 6, 3954-3962.

McDonald, N. R. 1998 The decay of cyclonic eddies by Rossby waves radiation. J. Fluid Mech. 361, 237-252.

McWilliams, J. C. \& Flierl, G. R. 1979 On the evolution of isolated, nonlinear vortices. J. Phys. Oceanogr. 9, 1155-1182.

Meid, R. P. \& Lindemann, G. J. 1979 The propagation and evolution of cyclonic Gulf Stream Rings. J. Phys. Oceanogr. 9, 1183-1206.

Pedlosky, J. 1987 Geophysical Fluid Dynamics, 2nd Edn. Springer.

Peng, M. S. \& Williams, R. T. 1990 Dynamics of vortex asymmetries and their influence on vortex motion on a $\beta$-plane. J. Atmos. Sci. 47, 1987-2003.

Rasmussen, J. J., Lynov, J. P., Hesthaven, J. S. \& Sutyrin, G. G. 1994 Vortex dynamics in plasma and fluids. Plasma Phys. Control. Fusion. 36, B193-B202. 
ReZnik, G. M. 1990 Motion of a point vortex on the $\beta$-plane. Oceanology. 30, 523-528.

RezniK, G. M. 1992 Dynamics of singular vortices on a beta-plane. J. Fluid Mech. 240, 405-432.

REZNIK, G. M. \& DEWAR, W. K. 1994 An analytical; theory of distributed axisymmetric barotropic vortices on the $\beta$-plane. J. Fluid Mech. 269, 301-321.

SMITH, R. K. \& UlRICH, W. 1990 An analytical theory of tropical cyclone motion using a barotropic model. J. Atmos. Sci. 47, 1973-1986.

Sutyrin, G. G. 1987 The beta-effect and the evolution of a localized vortex. Sov. Phys. Dokl. 32 791-793.

Sutyrin, G. G. 1988 Motion of an intense vortex on a rotating globe. Fluid Dyn. 23, 215-223.

Sutyrin, G. G. \& Flierl, G. R. 1994 Intense vortex motion on the beta-plane: Development of the beta gyres. J. Atmos. Sci. 51, 773-790.

Sutyrin, G. G., Hesthaven, J. S., Lynov, J. P. \& Rasmussen, J. J. 1994 Dynamical properties of vortical structures on the beta-plane. J. Fluid Mech. 268, 103-131.

Sutyrin, G. G. \& Morel, Y. G. 1997 Intense vortex motion in a stratified fluid on the beta-plane: an analytical theory and its validation. J. Fluid Mech. 336, 203-220.

YaO, H. B., Dritschel, D. G. \& Zabusky, N. J. 1995 High-gradient phenomena in two-dimensional vortex interactions. Phys. Fluids 7, 539-548. 